\title{
Dog-walking behaviours affect gastrointestinal parasitism in park-attending dogs
}

\author{
Anya F Smith', Christina AD Semeniuk², Susan J Kutz ${ }^{1}$ and Alessandro Massolo ${ }^{1 *}$
}

\begin{abstract}
Background: In urban parks, dogs, wildlife and humans can be sympatric, introducing the potential for inter- and intra-specific transmission of pathogens among hosts. This study was conducted to determine the prevalence of zoonotic and non-zoonotic gastrointestinal parasites in dogs in Calgary city parks, and assess if dog-walking behaviour, park management, history of veterinary care, and dog demographics were associated with parasitism in dogs

Methods: From June to September 2010, 645 questionnaires were administered to dog owners in nine city parks to determine behavioural and demographic factors, and corresponding feces from 355 dogs were collected. Dog feces were analyzed for helminth and some protozoan species using a modified sugar flotation technique and microscopic examination, a subsample was analyzed for Giardia spp. and Cryptosporidium spp. using a direct immunofluorescence assay. Descriptive and multivariate statistics were conducted to determine associations among behaviours, demographics, and parasite prevalence and infection intensities

Results: Parasite prevalence was 50.2\%. Giardia spp. (24.7\%), Cryptosporidium spp. (14.7\%), and Cystoisospora spp. $(16.8 \%)$ were the most prevalent parasites. Helminth prevalence was low (4.1\%). Presence of Giardia spp. was more likely in intact and young dogs; and infection with any parasite and Giardia spp. intensity were both positively associated with dogs visiting multiple parks coupled with a high frequency of park use and off-leash activity, and with being intact and young. Cryptosporidium spp. intensity was associated with being intact and young, and having visited the veterinarian within the previous year
\end{abstract}

Conclusions: Our results indicate a higher overall prevalence of protozoa in dogs than previously found in Calgary. The zoonotic potential of some parasites found in park-attending dogs may be of interest for public health. These results are relevant for informing park managers, the public health sector, and veterinarians.

Keywords: Dog, Gastrointestinal parasite, Zoonotic, Parasitism, Urban parks, Epidemiology

\section{Background}

Urban parks are a common destination for owners and their dogs [1]. Parks encourage a broad scope of healthy physical activity, including dog-walking [2], and are areas of socialization for dogs and their owners [3,4]. However, urban parks are also often confined areas where wildlife, dogs, and humans are sympatric, introducing the potential for disease transmission among domestic and wild animals. Environmental contamination and undisposed dog feces positive for zoonotic and non-zoonotic gastrointestinal (GI) parasites have been reported in urban

\footnotetext{
*Correspondence: amassolo@ucalgary.ca

'Department of Ecosystem and Public Health, Faculty of Veterinary Medicine, University of Calgary, 3280 Hospital Drive NW, Calgary, AB T2N 4Z6, Canada Full list of author information is available at the end of the article
}

parks [5-7], suggesting parks as potential sources of GI infection for dogs, humans, and wildlife.

Numerous studies investigating the epidemiology of GI parasites circulating in dogs have been conducted in urban areas worldwide. Demographics, geographic location, seasonal trends, and husbandry, have all been considered as risk factors for parasitism [5,8-10]. Potential risks for parasitism in dogs associated with park use have been investigated as well, although incomprehensively within multi-factor studies [5,8]. Only one study has focused solely on the direct association between park use and parasitism in dogs, and found a significant association between park attendance and Giardia spp. and Cryptosporidium spp. infections [11]. In Canada, few studies investigating GI infections in urban dogs 
have been carried out, and even fewer in Western Canada [5,12-14], despite that an estimated $33 \%$ of Canadians own dogs [15].

In Calgary, over 10,000 hectares of parkland are available for use by people and their dogs [16]. The potential for terrestrial and water contamination from wild and domestic canid feces, and from infected intermediate and paratenic rodent hosts in these parks introduces the possibility of infection in dogs. The potentially zoonotic parasites Echinococcus multilocularis, Toxocara canis, Ancylostoma caninum, Giardia spp., and nonzoonotic Toxascaris leonina and Uncinaria stenocephala, have been found in coyotes (Canis latrans) in Calgary [17-19]. With the exception of Taeniidae, these parasites have also been found in some shelter and homed dogs in Calgary [13], although potential associations to park use were not investigated. The occurrence of these parasites in homed dogs in Calgary [13] and the potential for increased exposure of park-attending dogs to sources of infection [11], prompted our assessment of enteric parasites in Calgary park-attending dogs.

This study focused on a subpopulation of urban dogs walked in city parks. In particular, we aimed to A) provide an inventory of enteric parasites in park-attending dogs in Calgary, and B) identify risk factors for dog parasitism associated with dog-walking behaviour in city parks, form of park management (on-leash, off-leash, or mixed off/on-leash), history of veterinary care, and dog demographics. We hypothesized urban park-walking to be a key risk factor for GI parasite infections in city dogs. In particular, we predicted the use of off-leash versus on-leash parks, the number of total parks visited, frequency of park use, and off-leash activity level within parks to be positively associated with dog parasitism.

\section{Methods}

\section{Study design and study areas}

We used an observational, cross-sectional study design. The study was conducted in Calgary, Alberta, Canada $\left(51^{\circ} 50 \mathrm{~N}, 114^{\circ} 55^{\prime} \mathrm{W}\right)$. Nine city parks were selected on the basis of park management and included three offleash, three on-leash, and three mixed management parks (parks with both on-leash and off-leash areas). Off-leash parks included Southland (SL), Nose Creek (NC) and Edworthy (EDW); on-leash parks were Stanley (SP), Fish Creek Provincial (FCPP), and Weaselhead (WSH); and mixed management sites were River (RP), Bowmont (BOW), and Nosehill (NH) Parks. All parks were used by coyotes.

\section{Interview protocol and questionnaire design}

Each park was visited once per week from June to September 2010. Dog owners were approached opportunistically in the park and asked to participate in a questionnaire and to provide their dogs' feces. A total of 635 surveys were conducted; an average of $71( \pm 4.1)$ per park.

The questionnaire consisted of 23 questions arranged within three sections: screening, dog demographics and human behaviour, and personal information. Dog demographic variables included breed, gender, age class, and spay or neuter status. Human behaviour variables included 1. Dog-walking behaviours: level of off-leash activity, park visitation frequency, whether the owner and dog visited one or greater than one park on a regular basis (park focus), and the number parks visited in addition to the most frequented park (number of additional parks; NAP); 2. Veterinary care history (whether or not the dog had visited the veterinarian in the previous year). Questions were open or close-ended. Off-leash activity was ranked on a four-point scale and ranged from "never" to "always". Visitation frequency was ranked on a six-point scale and ranged from "rarely" to "everyday" (Table 1). Research involving human subjects was approved by the University of Calgary Conjoint Faculties Research Ethics Board (file \#: 6498).

\section{Fecal sampling protocol}

Dog feces corresponding to questionnaires were opportunistically collected from owners at the end of their walk, or by requesting owners to collect a sample in a provided, labeled bag during their walk and deposit it into one of the coolers set up at select park exit points. A total of 550 samples corresponding to questionnaires were collected. The samples were kept at $-80^{\circ} \mathrm{C}$ for 72 hours to deactivate Echinococcus spp. eggs [20]. Samples were then transferred to $-20^{\circ} \mathrm{C}$ until laboratory analysis. The time interval between beginning of sample collection and laboratory analysis was approximately six months.

A subsample of 355 of the 550 samples collected was selected for laboratory analysis to achieve an average sample size of $39.4( \pm 0.47)$ samples per park and to deliberately maximize the number of dogs and owners that frequently attended parks, so as to determine the risk factors for parasitism specifically relevant to park attendees. Selection of cases for laboratory analysis were prioritized hierarchically according to the following criteria: 1) dogs with owners who showed a higher fidelity to one of the study sites than to parks not included in the study; 2) those with a single park focus; 3) those with a high visitation frequency to their most frequented park (MFP); 4) dogs 12 months of age or younger to maximize the juvenile contingent (Table 1). Selection using these criteria was made to ensure even sampling across parks, as well as including only one dog per household (i.e., if multiple dogs per household; the dog fulfilling the greatest number of criteria was chosen). Focusing on dog owners with an MFP equal to one of the 
Table 1 Survey design including questions, answer options, and scoring

\begin{tabular}{|c|c|c|}
\hline Section & Question & Answer \\
\hline \multicolumn{3}{|l|}{ 1. Screening } \\
\hline & Are you over the age of $18 ?$ & No $(0) / Y e s(1)$ \\
\hline & Is this your dog? & No $(0) / Y e s(1)$ \\
\hline & Does your dog normally defecate in this park? & No $(0) /$ Yes $(1)$ \\
\hline \multicolumn{3}{|c|}{ 2. Dog demographics and human behaviour } \\
\hline \multirow[t]{4}{*}{ Dog demographics } & What is the age of your dog? & Open \\
\hline & What is your dog's breed? & Mixed (1)/Purebred (2) \\
\hline & What is the gender of your dog? & Male (1)/Female (2) \\
\hline & Is your dog neutered or spayed? & No $(0) / Y e s(1)$ \\
\hline \multirow[t]{2}{*}{ Veterinary care history } & $\begin{array}{l}\text { Has your dog visited a veterinarian } \\
\text { within the last year? }\end{array}$ & No (0)/Yes (1)/Unknown (2) \\
\hline & $\begin{array}{l}\text { Have you de-wormed your dog in the last } 12 \\
\text { months (including heartworm medication)? }\end{array}$ & No (0)/Yes (1)/Unknown (2) \\
\hline \multirow[t]{10}{*}{ Walking behaviour } & How often do you come to this park? & 0 Rarely 0-3 Times/yr \\
\hline & & 1 Occasionally $<1 /$ mnth \\
\hline & & 2 Infrequently $1-3$ days/mnth \\
\hline & & 3 Regularly 1/week \\
\hline & & 4 Often 2-6 days/week \\
\hline & & 5 Everyday $1 /$ day \\
\hline & When do you come to this park? & Weekdays (1)/weekends (2)/both (3) \\
\hline & If a mixed park: management areas used & Off-leash (0)/On-leash (1)/both (2) \\
\hline & How often do you let your dog off-leash in this park? & Never (0)/Rarely (1)/Sometimes (2)/Always (3) \\
\hline & Do you visit any other parks in Calgary? & No $(0) / Y e s(1)$ \\
\hline \multirow[t]{11}{*}{ If yes: } & Which parks? & Open \\
\hline & $\begin{array}{l}\text { Which one of these additional parks do } \\
\text { you visit most often (P1)? }\end{array}$ & Open \\
\hline & How often do you go to this park (P1)? & 0 Rarely 0-3 Times/yr \\
\hline & & 1 Occasionally $<1 /$ mnth \\
\hline & & 2 Infrequently $1-3$ days/mnth \\
\hline & & 3 Regularly 1/week \\
\hline & & 4 Often 2-6 days/week \\
\hline & & 5 Everyday $1 /$ day \\
\hline & When do you go to this park? & Weekdays (1)/weekends (2)/both (3) \\
\hline & Park management type? & Off-leash (0)/On-leash (1)/both (2)/unknown (3) \\
\hline & How often do you let your dog off-leash in this park? & Never (0)/Rarely (1)/Sometimes (2)/Always (3) \\
\hline \multicolumn{3}{|l|}{ 3. Personal information } \\
\hline & What is your name? & Open \\
\hline & What is your postal code? & Open \\
\hline & What is your email address? & Open \\
\hline
\end{tabular}

nine study sites and who had a single park focus maximized representation of behavioural, demographic, and parasitic status across sites. Samples were prioritized to fulfill the highest number of selection criteria but it was not possible for all samples to fulfill all of the criteria. Once MFP and park focus were prioritized for those with an MFP equal to one of our nine study sites and a single park focus, and stratified into both prioritized and opposing groups, remaining samples were hierarchically selected in order of the highest to lowest ranks of park visitation frequency and, finally, selected for juvenile dogs. 


\section{Laboratory analysis}

Two sections of 2 grams of feces were removed from each fecal sample and analyzed separately for helminth eggs and coccidian oocysts using a modified Wisconsin double centrifugation technique [21] and subsequent microscope examination (Leika DME, 10X-40X). Intensity of eggs or oocysts per gram (epg or opg) was recorded and averaged between duplicate samples. Due to funding and time constraints, a subsample of 253 was selected from the 355 samples and investigated for Giardia spp. cysts and Cryptosporidium spp. oocysts. The subsample was selected to maximize equal representation of dogs across parks with an average of 28 per park $( \pm 0.20)$. Two grams of feces were concentrated and analyzed using a direct immunofluorescence assay (DFA) and a Fluorescein-labeled dual monoclonal antibody reagent (Waterborne Inc., New Orleans, Louisiana, USA). Cysts per gram (cpg) and opg were recorded.

Polymerase Chain Reaction (PCR) was performed following a described methodology of Multiplex-PCR for specific diagnosis of E. multilocularis, E. granulosus, and Taenia spp. [22]. Taenidae eggs collected from the fecal float of one dog sample (53 epg) were lysed in $50 \mu \mathrm{l}$ of DNA extraction buffer [ $500 \mathrm{mM} \mathrm{KCl,} 100 \mathrm{mM}$ Tris- $\mathrm{HCl}$ (pH 8.3), $15 \mathrm{mM} \mathrm{MgCl}_{2}, 10 \mathrm{mM}$ DTT, and 4.5\% Tween 20] containing $4 \mu \mathrm{l}$ of Proteinase K. Two $\mu \mathrm{l}$ of diluted lysate $(1: 20$ in $\mathrm{dH} 2 \mathrm{O})$ were used to run the multiplex-PCR. PCR products were electrophoresed on $2 \%$ agarose gel and visualized with UV light.

\section{Data analysis}

Dog owners using one of our nine study sites as their MFP were selected for descriptive, Chi-Square, and nonparametric analyses to ensure representation of dogs attending our study sites (sample $\mathrm{n}=345$; subsample $\mathrm{n}=$ 251). Estimated true parasite prevalence was calculated by incorporating parameters of imperfect screening tests [23] and median intensity were calculated overall, and stratified for dog age ( $\leq$ or $>12$ months old, juvenile and adult classes, respectively). Confidence intervals were also calculated using the Sterne estimator [24] and upper and lower limits calculated for intensity values. Median intensity values were rounded up to the nearest whole number. Helminth species were grouped together due to the low prevalence of individual species. Mean age of juvenile dogs was calculated to reflect the proportion of puppies ( $\leq 6$ months old) versus dogs older than six months comprising the juvenile age category. Chi-square exact test [25] was used to determine differences in occurrence of Giardia spp., Cryptosporidium spp., Cystoisospora spp., and helminths overall, within each age class and between age classes. Differences among median intensities of Giardia spp, Cryptosporidium spp., and Cystoisospora spp. between age classes were compared using Mann-Whitney Test for two independent samples [25].

To analyze the association between dog-walking behaviours or dog demographics and parasitism, we used logistic regression to predict presence or absence of different parasites, and linear regression to model infection intensity. Scores for off-leash activity and park visitation frequency in the MFP and second most frequented park (P1) were both pooled into two classes and summed (MFP + P1 values for off-leash activity or visitation frequency) to produce totals reflective of overall off-leash activity and visitation frequency. To accurately make this calculation, only dogs with owners whose MFP was the park where the questionnaire was conducted were included in analysis. MFP low frequency ranks of 0 and 1 (i.e., low fidelity to the selected parks) were filtered out to target park-attending dogs and to ensure the representation of the MFP (Table 1).

Principal Components Analysis for Categorical Data (CATPCA) [26] was performed to reduce the number of independent variables (behaviours, dog demographics and veterinary care history) and to control for correlated variables in multivariate analyses. CATPCA generates an additive model with as many dimensions as there are groups of tightly associated variables in the data [26]. Each dimension is an equation in which each variable has a coefficient (component loading) that represents its association with that dimension. Variables highly associated with each other have high loading magnitudes in the same component. The intention of the CATPCA was to summarize the high number of dog demographic and dog-walking behaviour variables; therefore, the form of park management was not included in the CATPCA. Two CATPCA dimensions were used in the multivariate models. The variables that did not enter into the CATPCA were entered into the multivariate models independently.

Binary logistic regression [27] was used to quantify relationships between the presence of any parasite (helminths or protozoa), of Giardia spp., of Cryptosporidium spp., or of Cystoisospora spp. using the CATPCA dimensions and the variables excluded from the CATPCA $(n=248)$. Presence of individual helminth species or genera and Sarcocystis spp. were not used as dependent variables due to their low prevalence values.

The relationship between parasite intensities (Cystoisospora spp., Giardia spp., and Cryptosporidium spp.), and the CATPCAs and variables were determined using negative binomial regression [27]. Only positive samples were used in the analysis, i.e. those with intensity values greater than zero (Cystoisospora spp. $\mathrm{n}=56$; Giardia spp. $\mathrm{n}=61$; Cryptosporidium spp. $\mathrm{n}=36$ ).

Statistical analyses were conducted using SPSS version 20.0 (SPSS, Chicago, Illinois, USA). 


\section{Results}

The overall prevalence of dogs infected with at least one parasite was $50.2 \%$. Juveniles comprised $15.9 \%$ of the sample. Prevalence of infection in this group was $70 \%$. Adult dogs comprised $84.1 \%$ of the sample and prevalence of infection was $46.4 \%$ (Table 2). The mean age of juveniles included in the sample (in months) was $9.34( \pm 0.37 ; n=55)$, and $9.30( \pm 0.41 ; n=40)$ in the subsample.

Giardia spp. (24.7\%), Cryptosporidium spp. (14.7\%), and Cystoisospora spp. (16.8\%) were the most prevalent parasites (Table 2). Prevalence of detected helminth species or genera was much lower overall (4.1\%) and included Capillaria spp. (1.4\%), Eucoleus spp. (0.60\%), Trichuris spp. (1.2\%), Toxascaris leonina (0.60\%), Toxacara canis (0.30\%), Taeniidae species (0.30\%), Diphylobothrium spp. (0.30\%), and Uncinaria stenocephala (0.30\%). Sarcocystis spp. was also detected $(0.30 \%)$.

PCR analysis resulted in amplicons of expected 117 and $267 \mathrm{bp}$ indicating positive diagnosis of E. granulosus and Taenia spp., respectively, and the absence of $395 \mathrm{bp}$ amplicons excluded the presence of E. multilocularis. We did not sequence the PCR products of E. granulosus and therefore could not confirm this diagnosis.

There were some significant differences in prevalence seen among Giardia spp., Cryptosporidium spp., Cystoisospora spp., and helminths overall, within each age class, and between age classes (Table 3). There was no significant difference between adults and juveniles in infection intensities of Giardia spp., Cystoisospora spp., or Cryptosporidium spp.

The final CATPCA analysis included off-leash activity total, park visitation frequency total, park focus, NAP, spay or neuter status, and age and resulted in two dimensions with Eigenvalues of $>1$, accounting for $78.1 \%$ of the total variance (Table 4). Breed, gender, and whether or not the dog had visited a veterinarian in the previous year, as well as park management type did not enter into the CATPCA. The first dimension (exposure) explained $54.5 \%$ of the total variance and grouped variables related to off-leash activity total, NAP, park focus, and park visitation frequency total. All variables in this dimension had large positive coefficients $(>0.6)$ with the exception of park focus, which was negatively associated with this dimension. The second dimension (dog demographics) explained $25.6 \%$ of the total variance and grouped dog demographic variables such as spay or neuter status and age that had positive high values $(>0.7)$ (Table 4).

The logistic models highlighted a statistically significant positive association between the presence of Giardia spp. and the dog demographics dimension (i.e. being juvenile and intact) (Table 5). Overall parasitism was significantly positively associated with exposure (off-leash activity and park visitation frequency totals, park focus, NAP) and dog demographics dimensions (Table 5).

Intensity of Giardia spp. infection was positively associated with exposure and dog demographics dimensions, as well as to additional demographics not incorporated in the CATPCA including female gender and mixed breed (Table 5). Cryptosporidium spp. intensity was significantly positively associated to the dog demographics dimension, as well as having visited the veterinarian in the previous year (Table 5).

\section{Discussion}

Our results indicated a higher prevalence of protozoa relative to helminths, and Giardia spp., Cystoisospora spp., and Cryptospordium spp. were the most prevalent parasites overall. Adults were parasitized significantly less often than juveniles, findings consistent with other studies $[8,9]$.

The dominance of protozoa versus helminth infection occurred overall and within both age classes. Higher infection rate with protozoa relative to helminths has also

Table 2 Prevalence (\%) and median intensity (n/g) of Gl parasites in dogs overall and stratified by age class

\begin{tabular}{|c|c|c|c|c|c|c|c|c|}
\hline \multirow[b]{2}{*}{ Age } & \multicolumn{2}{|c|}{ Giardia spp. } & \multicolumn{2}{|c|}{ Cryptosporidium spp. } & \multicolumn{2}{|c|}{ Cystoisospora spp. } & \multirow{2}{*}{$\begin{array}{c}\text { Helminths } \\
\%\end{array}$} & \multirow{2}{*}{$\begin{array}{c}\text { Overall } \\
\%\end{array}$} \\
\hline & $\mathbf{n} / \mathbf{g r}^{\mathbf{a}}$ & $\%$ & $\mathrm{n} / \mathrm{gr}$ & $\%$ & $\mathrm{n} / \mathrm{gr}$ & $\%$ & & \\
\hline \multirow[t]{3}{*}{ Juv } & $19.0^{\mathrm{b}}$ & 55.0 & 1.0 & 15.0 & 32.5 & 18.2 & 3.6 & 70.0 \\
\hline & $(1-600)^{c}$ & $(38.8-70.2)^{d}$ & $(1-200)$ & $(6.7-29.8)$ & $(1-250)$ & $(9.7-30.8)$ & $(.1-11.7)$ & $(53.8-82.9)$ \\
\hline & & $(n=40)$ & & $(n=40)$ & & $(n=55)$ & $(n=55)$ & $(n=40)$ \\
\hline \multirow[t]{3}{*}{ Adults } & 2.5 & 19.0 & 1.0 & 14.7 & 38.0 & 16.6 & 4.1 & 46.4 \\
\hline & $(1-500)$ & $(14.2-24.8)$ & $(1-10)$ & $(10.4-20.1)$ & $(1-250)$ & $(12.7-21.2)$ & $(2.0-7.1)$ & $(39.5-53.3)$ \\
\hline & & $(n=211)$ & & $(n=211)$ & & $(n=290)$ & $(n=290)$ & $(n=211)$ \\
\hline \multirow[t]{3}{*}{ Overall } & 6.0 & 24.7 & 1.0 & 14.7 & 38.0 & 16.8 & 4.1 & 50.2 \\
\hline & $(1-600)$ & $(19.7-30.5)$ & $(1-200)$ & $(10.7-19.7)$ & $(1-250)$ & $(13.2-21.1)$ & $(2.4-6.7)$ & $(44.0-56.4)$ \\
\hline & & $(n=251)$ & & $(n=251)$ & & $(n=345)$ & $(n=345)$ & $(n=251)$ \\
\hline
\end{tabular}

${ }^{\mathrm{a}}$ Number of cpg, opg, or epg $(\mathrm{n} / \mathrm{g})$.

${ }^{b}$ Median intensity values rounded up to the nearest whole number.

'Infection intensity intervals (upper and lower limits of epg, opg, or cpg).

${ }^{d} 95 \%$ confidence intervals. 
Table 3 Significant differences in parasite prevalence within each age class, between age classes, and overall

\begin{tabular}{|c|c|c|c|c|}
\hline & Juveniles $^{a}$ & Adults & Juveniles $>$ adults & Overall \\
\hline \multicolumn{5}{|l|}{ Giardia vs. Helminths } \\
\hline Prevalence (\%) & $55>3.6^{b}$ & $19>4.1$ & - & $24.7>4.1$ \\
\hline$x^{2}$ & 301.2 & 81.7 & - & 209.5 \\
\hline$d f$ & 1 & 1 & - & 1 \\
\hline P & $<0.001$ & $<0.001$ & - & $<0.001$ \\
\hline Giardia vs. Cryptosporidium spp. & & - & & \\
\hline Prevalence (\%) & $55>15$ & - & - & $24.7>14.7$ \\
\hline$x^{2}$ & 50.2 & - & - & 19.8 \\
\hline$d f$ & 1 & - & - & 1 \\
\hline P & $<0.001$ & - & - & $<0.001$ \\
\hline Giardia vs. Cystoisospora spp. & & - & & \\
\hline Prevalence (\%) & $55>18.2$ & - & - & $24.7>16.8$ \\
\hline$x^{2}$ & 36.5 & - & - & 11.5 \\
\hline$d f$ & 1 & - & - & 1 \\
\hline P & $<0.001$ & - & - & $=0.001$ \\
\hline \multicolumn{5}{|l|}{ Cryptosporidium spp. vs. Helminths } \\
\hline Prevalence (\%) & $15>3.6$ & $14.7>4.1$ & - & $14.7>4.1$ \\
\hline$x^{2}$ & 14.7 & 21 & - & 51.6 \\
\hline$d f$ & 1 & 1 & - & 1 \\
\hline P & $=0.003$ & $<0.001$ & - & $<0.001$ \\
\hline \multicolumn{5}{|l|}{ Cystoisospora spp. vs. Helminths } \\
\hline Prevalence (\%) & $18.2>3.6$ & $16.6>4.1$ & - & $16.8>4.1$ \\
\hline$x^{2}$ & 33.2 & 76.6 & - & 104 \\
\hline$d f$ & 1 & 1 & - & 1 \\
\hline$P$ & $<0.001$ & $=0.004$ & - & $<0.001$ \\
\hline \multicolumn{5}{|l|}{ Overall parasitism } \\
\hline Prevalence (\%) & - & - & $70>46.4$ & - \\
\hline$x^{2}$ & - & - & 8.9 & - \\
\hline df & - & - & 1 & - \\
\hline P & - & - & $<0.001$ & - \\
\hline
\end{tabular}

${ }^{a}$ Age strata. Significant differences in parasite prevalence within juvenile and adult age classes, between age classes (where parasite prevalence is higher in juveniles than adults), and overall. ' ${ }^{b}$ Example of coding for significant parasite prevalence differences. In this case, prevalence of Giardia spp. (55\%) is greater than helminths (3.6\%) in the juvenile age class.

been detected in other parts of the developed world [8]. This may be partially attributed to differences in abundance in the environment, degree of lifecycle complexity, and disease ecology among some protozoa and helminth species $[28,29]$. Veterinarians also typically treat for helminths more often than protozoa [30], another likely driver of this result.

Although the prevalence of helminths is clearly lower than protozoa in Calgary park-attending dogs overall, helminth prevalence is likely underestimated. The sensitivity of detecting certain helminth species using sucrose flotation is low [19], thus our use of sucrose flotation likely underestimated the true frequency of helminth infection in dogs. Other potential reasons for helminth prevalence underestimates include inability to detect prepatent infections, cyclical shedding of eggs [31-33], and hypobiosis [34]. This finding is particularly important in considering our potential detection of E. granulosus in a Calgary dog, and the recent discovery of E. multilocularis in coyotes [17-19], and T. canis in dogs [13] in the Calgary region. These parasites are zoonotic and the causative agents of potentially serious echinococcosis [35] and toxocariasis [36] in humans, respectively.

We detected a higher prevalence of Giardia spp. and Cryptosporidium spp. than recently found in dogs in Calgary [13]. Differences in the sensitivities of laboratory 
Table 4 Results of CATPCA

\begin{tabular}{lcc}
\hline & \multicolumn{2}{c}{ Component loadings } \\
Variable & \multicolumn{2}{c}{ Dimension } \\
\cline { 2 - 3 } & Exposure & Dog demographics \\
\hline Off-leash activity total & (2) \\
Park visitation frequency total & 1.029 & - \\
Park focus & -1.049 & - \\
Number of additional parks visited & 0.640 & - \\
Spay/neuter status & 0.142 & 0.919 \\
Age $\leq$ or $>12$ months & - & 0.856 \\
\hline
\end{tabular}

Component loading values represent a variable's association with each dimension. Variables highly associated with each other have high loading magnitudes in the same dimension.

techniques and in sampled populations are likely the reasons for the disparity. Joffe et. al. [13] investigated parasitism in a study population consisting primarily of homed dogs unscreened for park attendance, and accessed samples through veterinary clinics. We focused on a subpopulation of homed, park-attending dogs; dogs potentially more exposed to sources of infection than non-parkattending dogs, and therefore more likely parasitized.
Furthermore, the more sensitive DFA technique used here may partially account for the higher prevalence of Giardia spp. and Cryptosporidium spp. than zinc sulphate flotation used in the previous study [37]. However, our results also showed a higher prevalence of these two parasites in parkattending dogs than found by Wang et al. [11], who compared Giardia spp. and Cryptosporidium spp. prevalence in Colorado dogs that attended parks, versus dogs that did not attend parks, using an immunofluorescence technique similar to the one used in this study.

Giardia spp. was the most prevalent parasite overall and within both age classes. This is not surprising given it is one of the most common GI parasites infecting humans and domestic animals worldwide [38]. Giardia duodenalis is the only known species to infect both humans and dogs, but the degree of zoonotic risk is debated [39]. Currently, there are six species of Giardia and eight assemblages within the G. duodenalis species complex $[40,41]$. Several studies in North America confirmed assemblages $\mathrm{C}$ and $\mathrm{D}$ as the most common strains found to infect dogs, appearing largely host-specific, and nonzoonotic $[11,38]$. In addition to host-specific assemblages $\mathrm{C}$ and $\mathrm{D}$, assemblages with a broader host range such as $\mathrm{A}$ and $\mathrm{B}$ have also been found in dogs, although are more

Table 5 Significant results of two multivariate regression models: 1) A binary logistic regression model of the associations between GI parasite presence and dog demographic and exposure dimensions and age, breed, gender, and park management variables; 2) A negative binomial regression model of the associations between GI parasite median intensity and dog demographic and exposure dimensions and age, breed, gender, and park management variables

\begin{tabular}{|c|c|c|c|c|c|c|c|}
\hline \multicolumn{8}{|l|}{ Parameter } \\
\hline Estimates: & Factor & Wald Chi-Square & B & df & $P$ value & $L L$ & LL ratio $X^{2}$ \\
\hline \multicolumn{8}{|l|}{ 1) Presence/absence ${ }^{a}$ : } \\
\hline Giardia spp. $(n=248)$ & Dog Demographics Dimension & 15.2 & $-0.499^{b}$ & 1 & $<0.001$ & & \\
\hline Overall model & & & & 7 & 0.001 & -86.9 & 24.4 \\
\hline \multirow[t]{2}{*}{ Any parasite $(n=248)$} & Exposure Dimension & 4.9 & 0.287 & 1 & 0.026 & & \\
\hline & Dog Demographics Dimension & 8.3 & -0.366 & 1 & 0.004 & & \\
\hline Overall model & & & & 7 & 0.006 & -111.7 & 19.7 \\
\hline \multicolumn{8}{|l|}{ 2) Infection intensity: } \\
\hline \multirow[t]{2}{*}{ Giardia spp. $(n=61)$} & Exposure Dimension & 4.5 & $0.357^{c}$ & 1 & 0.035 & & \\
\hline & Dog Demographics & & & & & & \\
\hline \multirow[t]{3}{*}{ Dimension } & 7.0 & -0.278 & 1 & 0.008 & & & \\
\hline & Female Gender & 4.8 & 0.698 & 1 & 0.028 & & \\
\hline & Purebred & 3.9 & -0.620 & 1 & 0.048 & & \\
\hline Overall model & & & & 7 & 0.001 & -318.1 & 27.1 \\
\hline \multirow[t]{2}{*}{ Cryptosporidium spp. $(\mathrm{n}=36)$} & Dog Demographics Dimension & 11.4 & -0.751 & 1 & 0.001 & & \\
\hline & Visited Veterinarian within previous 12 months & 8.2 & 2.894 & 1 & 0.004 & & \\
\hline Overall model & & & & 7 & 0.001 & -87.9 & 48.8 \\
\hline
\end{tabular}

${ }^{a}$ The reference category for the dependant variable is 0 where $0=$ negative and $1=$ positive for parasites.

${ }^{b} \mathrm{~B}$ value magnitude and direction is the slope of the line and indicates the chance that a given dog has a value of 1 relative to the component loadings or independent variables.

${ }^{c} B$ value magnitude and direction is the slope of the line and indicates the direction of infection intensity of a given dog relative to the component loadings or independent variables. 
commonly seen in humans [42]. However, assemblagespecific infection in dogs can vary geographically, and potentially zoonotic assemblages A and B can even dominate as strains present in dogs in some regions of North America and Europe $[43,44]$. Coyotes have been reported to harbour assemblages A, D (Smith et al., unpublished data, [45]), and $\mathrm{C}$ in Alberta, and indicated as a possible reservoir for human infection in the province [45]. Direct transmission occurs among humans and animals via contaminated water, food, or physical contact [46]. Host species infected with Giardia spp. can be asymptomatic, or present clinical signs ranging from mild to severe diarrhea from malabsorption [47]. Giardia spp. is environmentally pervasive; the infective cysts are robust and can survive for long periods in cool, damp soil and water [40], both common park elements and potential sources of infection for park attending dogs. Cryptosporidium spp. and Cystoisospora spp. were also common protozoa found to infect park-attending dogs. Both parasites are directly transmitted and have a clinical presentation similar to Giardia spp. [46-48]. Like Giardia spp., Cryptosporidium spp. is a very common GI infectious agent worldwide [49], potentially zoonotic, with a robust infectious oocyst [50]. The Cryptosporidium genus is currently comprised of 19 species and over 40 genotypes. Cryptosporidium canis is the predominant species found in dogs $[43,51]$ and has also been found in coyotes in Alberta (Smith et al., unpublished data, [45]). Although C. canis is potentially zoonotic, it is considered very low risk to humans [51,52]. Human infections with Cryptosporidium are most often caused by host-specific Cryptosporidium hominis and less specific Cryptosporidium parvum [51]. C. parvum has infrequently been reported in dogs [51], but no evidence of infection in dogs with $C$. hominis has been detected in the literature. The appearance of Cryptosporidium spp. is an important finding; only two other recent Canadian GI parasite prevalence studies have detected this parasite in dogs, both reporting a lower prevalence than found here [53,54]. Cystoisospora spp. is non-zoonotic, but in extreme cases, can cause hemorrhagic gastroenteritis in dogs [48]. Although moderately prevalent, the overall median intensity was only 38 oocysts per gram. Clinical treatment is recommended for infection intensities at or above 1000 oocysts per gram [48], so concern for the impact of Cystoisospora spp. on park-attending dog health in Calgary appears minimal.

Our main hypothesis concerning intensity of park use and parasitism in dogs was supported: level of off-leash activity, number of parks visited, and frequency of park use, were shown to be positively associated to infection with at least one GI parasite species or genera. Behavioural risk factors for general parasitism were found to include [NAP], off-leash activity total, visiting more than one park, and frequency of park visitation. Dogs ranking high in these categories were more likely exposed to potential sources of infection including intermediate hosts, dog and coyote feces, and associated environmental contamination. The lack of detectable association between infection with Giardia spp., Cryptosporidium spp., or Cystoisospora spp. and park management and dogwalking behaviour may indicate a high level of occurrence of these parasites in all parks. With readily transmissible and environmentally resilient (oo)cysts, and as ubiquitous GI parasites found in dogs in general [40,52], a uniform level of exposure of dogs to these pathogens may exist across parks independent of park management or intensity of park use.

Giardia spp. was most likely to infect intact, juvenile dogs, findings also documented in past studies [8]. Giardia spp. and Cryptosporidium spp. infection intensities were also positively associated with being juvenile and intact. Giardia spp. intensity also showed a positive association with off-leash activity, visitation frequency, and number of parks visited, suggesting repeated exposure might contribute to elevated Giardia spp. cyst intensities in dogs. Further, Cryptosporidium spp. infection intensity was positively associated to visiting the veterinarian within the previous 12 months. Although our study did not explore whether or not infected dogs showed clinical signs, this result suggests that heavily infected dogs may have been symptomatic and subsequently visited the veterinarian.

One limitation of this study to note includes our subsample selection process. We used several criteria principally designed to include those participants who primarily attended one of our study sites and to maximize the number of frequent park users with high park fidelity who owned juvenile dogs, and therefore did not select park-attending dog owners and dogs at random. Although not all samples fulfilled all criteria, this selection process introduces selection bias and may inhibit internal validity to park-attending dogs. However, our intention was not to represent all park-attending dogs, but to determine demographic and behavioural risk factors for those park-attending dogs at a potentially higher risk for parasitism. We selected for juveniles in order to represent the few young dogs that attended parks and stratified for age accordingly. We aimed to represent these individuals across multiple park locales and forms of bylaw and provincial management, all in order to inform the management of high-risk behaviours. Also, we endeavoured to avoid respondent fatigue by surveying dog owners about select behaviours posing potential risks for parasitism in dogs, and did not attempt exhaustive data collection on this topic. We acknowledge other potential risk factors such as total number and species of animals in the home, tendency to drink from still water sources in parks etc. Lastly, we were not able to conduct an a priori power calculation due 
to the lack of published information on park-attending dog population size and estimated prevalence. Our prevalence estimates incorporated all available alternative data to improve estimated true prevalence such as estimates of sensitivity and specificity of our diagnostic tests, when known.

The outcomes of our study provide information for further epidemiological investigation and disease control interventions, and are beneficial to veterinarians for diagnostic purposes, park and wildlife managers, the public health sector, and dog owners. The positive associations found between intensity of park use and general parasitism, and Giardia spp. infection intensity, suggests that park attendance may pose a risk for parasitism in dogs. Prioritizing the removal of dog litter in parks is recommended to reduce and manage risk. Clearly, reducing the burden of dog feces in parks could have a positive health impact for dogs and humans by reducing infection risk, but cleaner parks may also offer an indirect health benefit by providing greater incentive to engage in physical and social activity. Dog litter left behind can be a deterrent to park use [2], and consequently, the accumulation of dog litter in some Calgary parks (Massolo et al., unpublished data) could discourage dog owners and non-dog owners from engaging in physical and social activity in these areas. Management strategies to reduce infection risk and promote park activity could include education campaigns highlighting the importance of picking up after dogs with respect to human, dog, and wildlife health, and increasing the frequency of dog feces cleanup in parks by city services. Veterinarians could also contribute to managing parasite transmission by performing regular examinations for protozoa in addition to helminths and educating clients about responsible park use.

Future research should focus on determining the public health significance of Giardia spp. and Cryptosporidium spp. infected dogs by conducting molecular studies to strain type these parasites. Also, clarifying the level of risk park attendance poses for GI parasitism in dogs is recommended by expanding the sample to 1) investigate Giardia spp. and Cryptosporidium spp. prevalence and strains in coyotes sympatric with park-attending dogs and; 2) include non-park-attending dogs as a reference group.

\section{Conclusions}

The prevalence of Giardia spp. and Cryptosporidium spp. in park-attending dogs is moderate to high. Certain behaviours within urban parks may contribute to parasitism in dogs, introducing potential implications for dog and human health. Public education and removal of dog feces is recommended in parks to maximize enjoyment and minimize parasite transmission risk.

\section{Competing interests}

The authors declare that they have no competing interests.

\section{Authors' contributions}

1) AS formulated the main research hypotheses, developed the questionnaire, conducted the fieldwork (including the collection of dog feces and administering of questionnaires), laboratory analysis, statistical analysis, and wrote the manuscript; 2) CS provided guidance in statistical analysis, and helped to revise the manuscript; 3) SK provided guidance in laboratory analysis, provided expertise in parasitology, and helped to revise the manuscript; 4) AM formulated the main research hypotheses, supervised and provided input in questionnaire development, fieldwork, statistical analysis, and provided substantial input in the development and design of the manuscript. All authors approved the final version of the manuscript.

\section{Acknowledgements}

We would like to thank Alistair Bath and Cormack Gates for assisting with project design and providing guidance in their areas of expertise. We also thank Stefano Liccioli for his collaboration on this project and Jesse Invik and Manigandan Lejeune Virapin and the Canadian Cooperative Wildlife Health Centre for help and support in the laboratory. This study was primarily funded by the National Sciences and Engineering Research Council of Canada, the Alberta Heritage Foundation for Medical Research Graduate Scholarships, and the University of Calgary, Faculty of Veterinary Medicine.

\section{Author details}

'Department of Ecosystem and Public Health, Faculty of Veterinary Medicine, University of Calgary, 3280 Hospital Drive NW, Calgary, AB T2N 4Z6, Canada. ${ }^{2}$ Great Lakes Institute for Environmental Research, University of Windsor, 401 Sunset Ave, Windsor, ON N9B 3P4, Canada.

Received: 2 May 2014 Accepted: 21 August 2014

Published: 4 September 2014

\section{References}

1. Macpherson $\mathrm{CN}$ : Human behaviour and the epidemiology of parasitic zoonoses. Int J Parasitol 2005, 35:1319-1331.

2. McCormack GR, Rock M, Toohey AM, Hignell D: Characteristics of urban parks associated with park use and physical activity: A review of qualitative research. Health Place 2010, 16:712-726.

3. Toohey A, Rock M: Unleashing their potential: a critical realist scoping review of the influence of dogs on physical activity for dog-owners and non-owners. Int J Behav Nutr Phys Act 2011, 8:1-9.

4. Westgarth C, Christley R, Pinchbeck G, Gaskell R, Dawson S, Bradshaw J: Dog behaviour on walks and the effect of the use of a leash. Appl Anim Behav Sci 2010, 125:38-46.

5. Gaunt MC, Carr AP: A survey of intestinal parasites in dogs from Saskatoon, Saskatchewan. Can Vet J 2011, 52:497-500.

6. Grimason A, Smith H, Parker J, Jackson M, Smith P, Girdwood R: Occurrence of Giardia spp. cysts and Cryptosporidium spp. oocysts in faeces from public health parks in the west of Scotland. Epidemiol Infect 1993, 110:641-645.

7. Habluetzel A, Traldi G, Ruggieri S, Attili AR, Scuppa P, Marchetti R, Menghini G, Esposito F: An estimation of Toxocara canis prevalence in dogs, environmental egg contamination and risk of human infection in the Marche region of Italy. Vet Parasitol 2003, 113:243-252.

8. Bugg RJ, Robertson ID, Elliot AD, Thompson RC: Gastrointestinal parasites of urban dogs in Perth, Western Australia. Vet J 1999, 157:295-301.

9. Fontanarrosa MF, Vezzani D, Basabe J, Eiras DF: An epidemiological study of gastrointestinal parasites of dogs from Southern Greater Buenos Aires (Argentina): age, gender, breed, mixed infections, and seasonal and spatial patterns. Vet Parasitol 2006, 136:283-295.

10. Little SE, Johnson EM, Lewis D, Jaklitsch RP, Payton ME, Blagburn BL, Bowman DD, Moroff S, Tams T, Rich L, Aucoin D: Prevalence of intestinal parasites in pet dogs in the United States. Vet Parasitol 2009, 166:144-152.

11. Wang A, Ruch Gallie R, Scorza V, Lin P, Lappin MR: Prevalence of Giardia and Cryptosporidium species in dog park attending dogs compared to non-dog park attending dogs in one region of Colorado. Vet Parasitol 2012, 184:335-340.

12. Anvik J, Hague A, Rahaman A: A method of estimating urban dog populations and its application to the assessment of canine fecal pollution and endoparasitism in Saskatchewan. Can Vet J 1974, 15:219-223. 
13. Joffe D, Van Niekerk D, Gagne F, Gilleard J, Kutz S, Lobingier R: The prevalence of intestinal parasites in dogs and cats in Calgary, Alberta. Can Vet J 2011, 52:1323-1328.

14. Lewis P: Prevalence of Giardia sp. in dogs from Alberta. In Advances in Giardia Research. Edited by Wallis P, Hammond B. Alberta, Canada: University of Calgary Press; 1988:61-64.

15. Perrin $\mathrm{T}$ : The business of urban animals survey: the facts and statistics on companion animals in Canada. Can Vet J 2009, 50:48-52.

16. City of Calgary Parks Webpage. http://www.calgary.ca/CSPS/Parks/Pages/ home.aspx?redirect=/parks.

17. Catalano S, Lejeune M, Liccioli S, Verocai GG, Gesy KM, Jenkins EJ, Kutz SJ, Fuentealba C, Duignan PJ, Massolo A: Echinococcus multilocularis in urban coyotes, Alberta, Canada. Emerg Infect Dis 2012, 18:1625-1628.

18. Liccioli S, Catalano S, Kutz S, Lejeune M, Verocai G, Duignan P, Fuentealba C, Hart M, Ruckstuhl K, Massolo A: Gastrointestinal parasites of coyotes (Canis latrans) in the metropolitan area of Calgary, Alberta, Canada. Can J Zool 2012, 90:1023-1030.

19. Liccioli S, Catalano S, Kutz SJ, Lejeune M, Verocai G, Duignan PJ, Fuentealba C, Ruckstuhl K, Massolo A: Sensitivity of double centrifugation sugar fecal flotation for detecting intestinal helminths in coyotes (Canis latrans). J Wildl Dis 2012, 48:717-723.

20. Veit P, Bilger B, Schad V, Schafer J, Frank W, Lucius R: Influence of environmental factors on the infectivity of Echinococcus multilocularis eggs. Parasitol 1995, 110(Pt 1):79-86.

21. Cox DD, Todd AC: Survey of gastrointestinal parasitism in Wisconsin dairy cattle. J Am Vet Med Assoc 1962, 141:706-709.

22. Trachsel $D$, Deplazes $P$, Mathis $A$ : Identification of taeniid eggs in the faeces from carnivores based on multiplex PCR using targets in mitochondrial DNA. Parasitology 2007, 134:911-920.

23. Rogan WJ, Gladen B: Estimating prevalence from the results of a screening test. Am J Epidemiol 1978, 107:71-76.

24. Santner TJ, Duffy DE: The Statistical Analysis of Discrete Data. New York: Springer; 1989

25. Good P: Permutation, Parametric, and Bootstrap Tests of Hypotheses. 3rd edition. New York: Springer Science and Business Media Inc.; 2005

26. Kaplan D: The Sage Handbook of Quantitative Methodology for the Social Sciences. Thousand Oaks, Cali: Sage Publications; 2004.

27. Fox J: Applied Regression Analysis and Generalized Linear Models. 2nd edition. Los Angeles: Sage Publications; 2008.

28. Churcher TS, Ferguson NM, Basanez MG: Density dependence and overdispersion in the transmission of helminth parasites. Parasitology 2005, 131:121-132

29. Hudson P, Rizzoli A, Grenfell B, Heesterbeek H, Dobson A: The Ecology of Wildlife Diseases. New York, USA: Oxford University Press; 2001.

30. Companion Animal Parasite Council Recommendations for Parasite Prevention and Control. http://www.capcvet.org/capc-recommendations/.

31. Conboy G: Helminth parasites of the canine and feline respiratory tract. Vet Clin North Am-Small Animal Practice 2009, 39:1109-1126.

32. Conboy G: Cestodes of dogs and cats in North America. Vet Clin North Am-Small Animal Practice 2009, 39:1075-1090.

33. Epe C: Intestinal nematodes: biology and control. Vet Clin North Am-Small Animal Practice 2009, 39:1091-1107.

34. Gibbs HC: Mechanism of survival by nematode parasites with emphasis on hypobiosis large-scale management-systems and parasite population. Magyar Allatorvosok Lapja 1982, 37:765-769.

35. Eckert J, Conraths FJ, Tackmann K: Echinococcosis: an emerging or re-emerging zoonosis? Int J Parasitol 2000, 30:1283-1294.

36. Despommier D: Toxocariasis: clinical aspects, epidemiology, medical ecology, and molecular aspects. Clin Microbiol Rev 2003, 16:265-272.

37. Veronesi F, Passamonti F, Caccio SM, Diaferia M, Piergili Fioretti D: Epidemiological survey on equine Cryptosporidium and Giardia infections in Italy and molecular characterization of isolates. Zoonoses Public Health 2010, 57:510-517.

38. Xiao L, Fayer R: Molecular characterisation of species and genotypes of Cryptosporidium and Giardia and assessment of zoonotic transmission. Int J Parasitol 2008, 38:1239-1255.

39. Bowman DD, Lucio-Forster A: Cryptosporidiosis and giardiasis in dogs and cats: veterinary and public health importance. Exp Parasitol 2010, 124:121-127.

40. Feng Y, Xiao L: Zoonotic potential and molecular epidemiology of Giardia species and giardiasis. Clin Microbiol Rev 2011, 24:110-140.
41. Lasek-Nesselquist $E$, Welch DM, Sogin ML: The identification of a new Giardia duodenalis assemblage in marine vertebrates and a preliminary analysis of $\mathrm{G}$. duodenalis population biology in marine systems. Int J Parasitol 2010, 40:1063-1074

42. Ballweber LR, Xiao L, Bowman DD, Kahn G, Cama VA: Giardiasis in dogs and cats: update on epidemiology and public health significance. Trends Parasitol 2010, 26:180-189.

43. Thompson RC, Palmer CS, O'Handley R: The public health and clinical significance of Giardia and Cryptosporidium in domestic animals. Vet $J$ 2008, 177:18-25.

44. Covacin C, Aucoin DP, Elliot A, Thompson RC: Genotypic characterisation of Giardia from domestic dogs in the USA. Vet Parasitol 2011, 177:28-32.

45. Thompson RC, Colwell DD, Shury T, Appelbee AJ, Read C, Njiru Z, Olson ME: The molecular epidemiology of Cryptosporidium and Giardia infections in coyotes from Alberta, Canada, and observations on some cohabiting parasites. Vet Parasitol 2009, 159:167-170.

46. Hunter PR, Thompson RC: The zoonotic transmission of Giardia and Cryptosporidium. Int J Parasitol 2005, 35:1181-1190.

47. Buret A: Pathogenic Mechanisms in Gardiasis and Cryptosporidiosis. In Giardia and Cryptosporidium: From Molecules to Disease. Edited by Ortega Pierres G, Caccio S, Fayer R, Mank T, Smith H, Thompson R. Oxfordshire, United Kingdom: CABl; 2009:428-441.

48. Foreyt W: Veterinary Parasitology Reference Manual. 5th edition. Ames, lowa: lowa State University Press; 2001.

49. Heitman TL, Frederick LM, Viste JR, Guselle NJ, Morgan UM, Thompson RC, Olson ME: Prevalence of Giardia and Cryptosporidium and characterization of Cryptosporidium spp. isolated from wildlife, human, and agricultural sources in the North Saskatchewan River Basin in Alberta, Canada. Can J Microbiol 2002, 48:530-541.

50. Fayer R, Morgan U, Upton SJ: Epidemiology of Cryptosporidium: transmission, detection and identification. Int J Parasitol 2000, 30:1305-1322.

51. Lucio-Forster A, Griffiths JK, Cama VA, Xiao L, Bowman DD: Minimal zoonotic risk of cryptosporidiosis from pet dogs and cats. Trends Parasitol 2010, 26:174-179.

52. Xiao L: Molecular epidemiology of cryptosporidiosis: an update. Exp Parasitol 2010, 124:80-89.

53. Blagburn BL, Schenker R, Gagne F, Drake J: Prevalence of intestinal parasites in companion animals in Ontario and Quebec, Canada, during the winter months. Vet Ther 2008, 9:169-175.

54. Bryan HM, Darimont CT, Paquet PC, Ellis JA, Goji N, Gouix M, Smits JE: Exposure to infectious agents in dogs in remote coastal British Columbia: Possible sentinels of diseases in wildlife and humans. Can $J$ Vet Res 2011, 75:11-17

\section{doi:10.1186/1756-3305-7-429}

Cite this article as: Smith et al: Dog-walking behaviours affect gastrointestinal parasitism in park-attending dogs. Parasites \& Vectors 2014 7:429.

\section{Submit your next manuscript to BioMed Central and take full advantage of:}

- Convenient online submission

- Thorough peer review

- No space constraints or color figure charges

- Immediate publication on acceptance

- Inclusion in PubMed, CAS, Scopus and Google Scholar

- Research which is freely available for redistribution 